Cahiers de civilisation médiévale

\title{
Collectif, Le beau et la beauté au Moyen Âge
}

\section{Marie-Pascale Halary}

\section{OpenEdition}

\section{Journals}

Édition électronique

URL : https://journals.openedition.org/ccm/5373

DOI : $10.4000 / \mathrm{ccm} .5373$

ISSN : 2119-1026

\section{Éditeur}

Centre d'études supérieures de civilisation médiévale/Université de Poitiers

\section{Édition imprimée}

Date de publication : 1 décembre 2020

Pagination : $270-273$

ISBN : 978-2-490783-07-6

ISSN : 0007-9731

Référence électronique

Marie-Pascale Halary, "Collectif, Le beau et la beauté au Moyen Âge », Cahiers de civilisation médiévale [En ligne], 252 | 2020, mis en ligne le 02 décembre 2020, consulté le 23 novembre 2022. URL : http:// journals.openedition.org/ccm/5373 ; DOI : https://doi.org/10.4000/ccm.5373

Creative Commons - Attribution - Pas d'Utilisation Commerciale - Pas de Modification 4.0 International - CC BY-NC-ND 4.0

https://creativecommons.org/licenses/by-nc-nd/4.0/ 
Le beau et la beauté au Moyen Âge, O. BoulnoIs et I. Moulin (dir.), Paris, Librairie philosophique J. Vrin, 2018.

Cet ouvrage, issu d'un colloque qui s'est tenu en novembre 2011 dans le cadre de l'Institut d'études médiévales de l'Institut catholique de Paris, s'intéresse au concept de beauté au Moyen Âge : reprenant et prolongeant selon diverses modalités les réflexions qu'Olivier Boulnois a proposées dans un article important («La beauté d'avant l'art: d'Umberto Eco à saint Thomas d'Aquin, et retour», dans Le Souci du passage. Mélanges offerts à Jean Greisch, P. CAPELLEDumont, G. Hébert et M.-D. Popelard [dir.], Paris, Les Éditions du Cerf [Philosophie et théologie], 2004, p. 414-442), il interroge la pertinence de la catégorie d'esthétique pour la conception médiévale du beau. Comme le rappelle l'introduction $(\mathrm{O}$. Boulnois, «Dire la beauté au Moyen Âge : les paroles et les œuvres», p. 11-15), est-il possible de parler d'esthétique i.e. d'accorder une priorité au sensible quand la beauté est d'abord intelligible? L'ouvrage s'inscrit ainsi dans le prolongement critique de plusieurs textes fondamentaux sur la question de la beauté, en particulier ceux d'Edgar de Bruyne (Études d'esthétique médiévale suivi de L'Esthétique au Moyen Age Paris, Albin Michel [Bibliothèque de l'évolution de l'humanité, 29-30], 1998 [1946], 2 vol.) et d'Umberto Eco (notamment Le Problème esthétique chez Thomas d'Aquin M. JAVION [trad.], Paris, Presses universitaires de France [Formes sémiotiques], 1993 [1970]). Examinant aussi bien les textes théoriques que les œuvres artistiques, le livre s'organise autour de deux perspectives que l'introduction appelle respectivement «l'ordre des discours» (p. 11) et les «œuvres d'art elles-mêmes» (p. 13).

Le premier ensemble de contributions analyse des textes philosophiques et théologiques et permet de repérer, à défaut d'une esthétique, une véritable pensée de la beauté. O. Boulnois commence par revenir sur l'anachronisme de la conception, moderne, d'esthétique («De l'esthétique médiévale, derechef, qu'elle n'existe pas», p. 17-38) : il réfute successivement les trois arguments parfois avancés en faveur d'une «esthétique médiévale» (le beau comme transcendantal, l'identification de l'art aux beauxarts et l'inscription du beau dans la seule perception sensible) et montre qu'au Moyen Âge, la beauté, avant de se manifester dans l'art, est un attribut du divin. De ce fait, les beautés sensibles, qui peuvent être ressemblantes, ne sont pas désirées pour ellesmêmes (ce qui est constitutif de l'esthétique telle qu'elle a été définie par Emmanuèle Baumgarten) : ce qui caractérise le Moyen Âge, c'est donc «une anti-esthétique, ou une esthétique théologique» (p. 33). Deux éléments particulièrement intéressants sont à signaler. L'analyse, tout d'abord, apporte par sa dimension méthodologique et épistémologique : on le sait, le Moyen Âge impose d'historiciser les catégories, de mesurer que certaines d'entre elles sont appliquées de manière rétrospective et anachronique. La solution proposée ici recourt à la catégorie foucaldienne d'épistémè : ce n'est pas parce qu'on trouve des jugements de goût au Moyen Âge ou des discours sur le beau et sur l'art que ceux-ci se rencontrent dans une épistémè commune (ou une esthétique). Autrement dit, à l'instar d'une formule de Thomas d'Aquin souvent citée pour essayer de montrer l'existence d'une théorie transcendantale du beau chez celui-ci, il faut inscrire les discours et les pensées dans l'épistémè qui était la leur. D'autre part, l'a. invite à réfléchir à ce que le Moyen Âge «fait»à notre conception du beau. Au fond, dit-il en substance, 
l'exemple médiéval permet de penser la beauté hors d'une esthétique, laquelle apparaît de ce fait comme une catégorie non nécessaire et historique : «le monde d'après l'art, dans lequel nous vivons, peut renouer avec la beauté d'avant l'art» (p. 20).

Après cette étude de philosophie générale, plusieurs contributions présentent des auteurs et des textes, classés chronologiquement, qui constituent d'importants jalons dans la conception médiévale de la beauté. Le premier de ces jalons est le Phèdre de Platon : Anca Vasiliu, dans une enquête intitulée «Comment parler du beau? La réponse de Platon dans le Phèdre et son interprétation par Plotin» (p. 39-74) interroge le sens de ce texte à partir de sa réception postérieure et, notamment, néoplatonicienne. L'a. montre comment la lecture du Banquet a parfois infléchi l'interprétation du Phèdre et, étudiant les rapports entre kallos et logos, elle souligne les pouvoirs déictiques du langage face à la beauté, «seule forme intelligible qui se voit» (p. 69). La spécificité de cette Idée qui s'offre à la vue ne semble pas perçue par Plotin, dont la conception du beau se rapproche de celle de Diotime et qui se ferme au visible pour accéder à la beauté. Kristina Mitalaité, ensuite, s'intéresse à deux discours carolingiens sur la beauté à partir de la question de l'image artificielle («L'image peut-elle être belle? L'image matérielle de la conception de la beauté chez les Carolingiens [fin VIII'-début IX ${ }^{\mathrm{e}}$ s.]», p. 75-92). Théodulfe d'Orléans, dans le traité de polémique lié au concile Nicée II, l'Opus Caroli regis contra synodum, procède à une condamnation de l'image, réduite à un simple ornamentum, qui occulte la vérité de la foi et risque de devenir l'objet d'un culte idolâtre. Dans le In honorem sanctae crucis, Raban Maur, au contraire, établit la possibilité de la figuration de la croix en se fondant sur la forme imitable qu'est le Christ. La beauté mathématique de cette figure, qu'il exprime par la géométrie autant que par les louanges, offre alors une représentation parfaite de la foi. De l'image artificielle dans le monde carolingien, l'article de Dominique Poirel permet de passer à la beauté des créatures et de Dieu chez Hugues de Saint-Victor ( Dieu, la nature et l'homme : la place de la beauté dans l'œuvre d'Hugues de Saint-Victor», p. 93-121). L'a. propose un parcours à travers quatre traités (le Detribus diebus, le commentaire sur la Hiérarchie céleste, le De arrha animae et le De institutione nouitiorum) afin de montrer que, chez le victorin, la beauté est non seulement un thème central mais qu'elle est surtout au cœur de la vie spirituelle. Si la beauté des créatures suscite une véritable admiration, c'est que, outre les caractéristiques qu'elle possède, cette beauté rayonne de la sagesse de Dieu. La pulchritudo divine est en effet forme (au sens de modèle) et «formifique», productrice. Tout un parcours de beauté est alors proposé pour la créature humaine : la beauté est objet de contemplation et d'amour et la splendeur originelle peut être restaurée en l'homme. C'est en cela qu'elle possède une dimension spirituelle : le retour à la beauté signale le rétablissement de l'image et de la ressemblance. S'intéressant à Bonaventure, Laure Solignac dans son étude «Saint Bonaventure et le "Fils très beau" : naissance d'un transcendantal approprié» (p. 123-145) revient sur l'inscription du pulchrum parmi les transcendantaux, question déjà évoquée par O. Boulnois. D'une part, l'a. réfléchit aux implications que la nature transcendantale du beau pourrait avoir sur l'émergence d'une esthétique : la reconnaissance de cette nature contribuet-elle à un progrès duquel participe l'apparition d'une discipline autonome consacrée à la beauté ou alors l'identification du beau comme transcendantal constitue-t-elle un obstacle à l'établissement d'une esthétique? D'autre part, cette contribution revient sur les évolutions entre un brouillon attribué à Bonaventure, la Summa Halensis et différents textes bonaventuriens (en particulier le Commentaire des Sentences) : alors que le pulchrum est promu au rang de transcendantal dans le premier texte, ce n'est plus le cas ensuite. Mais «cette destitution s'accompagne de la plus belle promotion» (p. 135) : approprié à la personne du Fils, le beau permet désormais d'explorer la vie trinitaire. Après un article en allemand sur la théorie du beau chez Albert le Grand (Henryk Anzulewicz, "Strukturelemente der neuplatonisch-Dionysischen Theorie des Schönen bei Albertus Magnus», p. 147-176), ces études des discours sur la beauté se terminent par une contribution d'Olivier-Thomas Vénard : "Y a-t-il un problème esthétique chez saint Thomas d'Aquin?» (p. 177-200). L'a. présente une analyse critique de la lecture de l'esthétique thomasienne par U. Eco qui, dans l'ouvrage déjà cité, voit dans la conception de la beauté de Thomas un "problème esthétique». O.-T. Vénard montre que le spécialiste italien perçoit une aporie parce qu'il réduit la pensée de Thomas à un système philosophique. Mais Thomas est d'abord un maître en sacra pagina: la beauté est avant tout celle de Dieu et ressortit à la théologie plus qu'à une esthétique.

Les études qui suivent portent, non sur des discours relatifs à la beauté, mais sur de belles œuvres. Plusieurs articles sont consacrés à l'architecture et à la sculpture. C'est d'abord le cas de «La beauté monumentale» de Florian Meunier (p. 201-212). Revenant sur l'anachronisme de catégories comme 
esthétique, roman, gothique, l'a. s'interroge sur les critères qui conditionnent le jugement de beauté au Moyen Âge. Dans une société qui distingue arts libéraux et arts mécaniques, la «fabrique» de la beauté monumentale semble très largement laissée à l'appréciation de ceux que nous appelons aujourd'hui des artistes. Passant des œuvres architecturales à un ensemble textuel parfois lu comme le manifeste de l'art gothique, Andreas Speer s'intéresse aux écrits de Suger («L'esthétique médiévale comme expérience de l'art : les écrits de l'abbé Suger à Saint-Denis », p. 213-226). Il montre que l'abbé de Saint-Denis fait l'objet d'une construction et que le modèle explicatif communément reçu, en raison notamment d'Erwin Panofsky (Architecture gothique et pensée scolastique, Pierre Bourdieu [trad.], Paris, Éditions de Minuit [Le sens commun], 1967), rapporte les choix architecturaux à une «pensée métaphysique» (p. 222). En réalité, montre l'a., le programme architectural se comprend bien plus si on repère la volonté de Suger de respecter la vieille basilique de Dagobert et d'ordonner un espace véritablement liturgique. L'enquête sur la beauté de Saint-Denis se poursuit avec l'article de Dominique Alibert («De terre et d'or, de bois et de lumière : Suger et la reconstruction de Saint-Denis », p. 227-242) : partant de l'opposition bien connue entre les propos de Bernard de Clairvaux sur l'ornementation et ceux de Suger, l'a. interroge le sens de certains développements du De administratione consacrés à la richesse. Il montre que ce déploiement de matières précieuses s'explique par le projet de faire de l'abbatiale « une ébauche terrestre de la cité céleste» (p. 231-232). Sur les rapports entre Bernard et Suger autant que sur le sens et les enjeux de la circulation des richesses dans les textes de Suger, différentes études auraient pu être exploitées, en particulier Jean-Claude BonNe, «Pensée de l'art et pensée théologique dans les écrits de Suger», dans Artistes et philosophes, éducateurs?, Ch. Descamps (dir.), Paris, Centre Georges-Pompidou (Espace international, philosophie), 1994, p. 13-50; André MoISAN, «Suger de Saint-Denis, Bernard de Clairvaux et la question de l'art sacré», dans Le Beau et le Laid au Moyen Âge, Aix-en-Provence, CUERMA/Université de Provence (Sénéfiance, 43), 2000, p. 383-399 et Éléonore ANDRIEU, «Le regard stupéfait, la beauté et la mise en ordres dans les textes de Suger», dans La Beauté du merveilleux, A. GAILlard et J.-R. VAlette (dir.), Bordeaux, Presses universitaires de Bordeaux (Mirabilia), 2011, p. 111-141.

Après ces études sur la beauté monumentale, François Cassingena-Trévedy s'intéresse aux critères de la beauté sonore dans la monodie romano-franque
(«"Speciosus forma” [Ps XLIV, 3] : l'enluminure sonore des mots qui disent la beauté dans le répertoire du chant romano-franc», p. 243-263). Si la beauté de ce qui est chanté tient à la reprise de la Parole scripturaire, la splendeur du chant lui-même s'explique par certaines qualités telles la suavitas. L'a. termine en soulignant la beauté des anciens manuscrits et de la notation neumatique : tout concourt à une «illustration visuelle et sonore de la Parole de Dieu» (p. 263). Félicité Schuler-Lagier, quant à elle, analyse «Le langage symbolique dans les images médiévales de Chartres » (p. 265-306). Elle commence par des développements généraux et un peu longs sur la lecture des images médiévales; ce n'est que dans les dernières pages (p. 304-306) qu'elle aborde plus frontalement la question de la laideur et de la beauté des images se trouvant sur les vitraux de Chartres.

Un bel article de Philippe Sers («Beauté et vérité : l'expérience spirituelle dans l'icône byzantino-slave», p. 307-319) permet d'ouvrir un volet consacré à l'art byzantin. Dans cette première étude, l'a. interroge l'étrangeté de la beauté de l'icône et montre que les modalités de la représentation font que cette image réfère à une icône archétypale. L'écriture de l'icône, au demeurant, est permise par la prière et une rencontre spirituelle : la beauté du Christ est offerte à celui qui l'accueille, tout comme la vérité de la Parole divine. Grégoire Aslanoff clôt cette partie avec un article intitulé "“Sans beauté ni éclat" (Is 53, 2) : la représentation du Christ mort dans l'art byzantin » (p. 321-331) - que, malheureusement, 1'introduction n'annonce pas. G. Aslanoff se demande en quoi consiste la beauté du Dieu crucifié. Après être revenu sur les différentes modalités de la figuration du Christ de la Passion, il montre que, pour l'Église orthodoxe, qui relie la Crucifixion à la Résurrection, la valeur de cette représentation tient à l'association de la mort et de la vie.

L'ouvrage se termine par une conclusion, efficace, due à Isabelle Moulin («Postface : esthétique et Kalokagathie. Aux sources du beau et de la beauté», p. 333-339), suivie d'un index nominum (p. 341-347), d'une liste des illustrations (p. 349-350) et d'une liste des contributeurs (p. 351-352).

L'ensemble se caractérise par une mise en page très soignée : seules de rares coquilles ont été repérées, ainsi qu'une erreur dans la numérotation et le référencement des illustrations relatives à l'article de F. Meunier. On peut toutefois regretter l'absence de bibliographie générale. En outre, il aurait été intéressant qu'une étude soit consacrée à la beauté rhétorique : si la valeur de l'ornementation verbale 
est parfois évoquée (p. 76 et p. 94 par ex.), la partie portant sur l'ordre des discours aurait pu intégrer une analyse de la beauté littéraire telle qu'elle est définie par les arts poétiques : voir en ce sens JeanYves Tilliette, Des mots à la parole : une lecture de la Pœtria nova de Geoffroy de Vinsauf, Genève, Droz (Recherches et Rencontres, 16), 2000.

Au-delà de ces deux regrets, les apports de l'ouvrage sont incontestables. Dans le prolongement de l'article d'O. Boulnois déjà cité, il propose de poser autrement la question de la beauté au Moyen Âge, hors d'une esthétique, c'est-à-dire en s'émancipant de la catégorie anachronique favorisée par les études fondatrices que furent celles d'E. de Bruyne et d'U. Eco. Comme le note d'ailleurs la conclusion, la naissance de l'esthétique au XVIII ${ }^{\mathrm{e}} \mathrm{s}$. marque une rupture avec un autre régime de la beauté, la kalokagathie, régime défini par l'unité entre le beau et le bien. C'est cette historicité que l'ensemble de ce livre invite à ressaisir - et cette approche aurait pu davantage être signalée dans le titre général ou dans un éventuel sous-titre.

Outre qu'il fait cette proposition très stimulante, l'ouvrage constitue à bien des égards une véritable synthèse critique des principales études que le $\mathrm{XX}^{\mathrm{e}} \mathrm{s}$. a consacrées à la beauté médiévale : des contributions reviennent non seulement sur les travaux d'E. de Bruyne et d'U. Eco mais aussi sur ceux d'E. Panofsky, d'Étienne Gilson ou de Hans Urs Von Balthasar.

Une autre force de cet ouvrage tient à sa dimension résolument pluridisciplinaire et comparatiste (les développements sur le monde byzantin sont intéressants). De véritables dialogues s'établissent entre les différents spécialistes (sur la nature transcendantale du beau et les rapports entre le beau et le bon, sur les textes de Suger, sur la beauté de la Croix), ce que l'organisation des différents articles met en valeur (mais cette architecture, bien pensée, aurait gagné à être explicitée grâce à des subdivisions).

L'ensemble de cet ouvrage est de très bonne tenue, avec plusieurs contributions importantes à la réflexion sur la beauté médiévale.

Marie-Pascale HALARY

UMR 5648 - Ciham

Université Lumière Lyon 2 